\title{
Spacial Simulation of Shipboard Operations for Skid-equipped Rotary-wing Aircraft
}

\author{
Alexander Schock \\ MASc. Candidate \\ Carleton University \\ Ottawa, Canada \\ AlexRSchock@cmail.carleton.ca
}

\author{
Robert Langlois \\ Dept. of Mechanical and Aerospace Engineering \\ Carleton University \\ Ottawa, Canada \\ Robert.Langlois@carleton.ca
}

\begin{abstract}
SRAMSS (Skid-equipped Rotary-wing Aircraft Manoeuvring and Securing Simulation) is a simulation package aimed at generating transient aircraft behavior to study the dynamic interface between skid-equipped rotary-wing aircraft and ship decks during shipboard operations in elevated sea and weather conditions. The package incorporates dynamic finite element modelling into Kane's method for dynamics equations, contact mechanics with oriented dynamic response including the LuGre friction model to capture complex friction behavior, aerodynamic drag, and blade element theory to generate induced thrust and moments from the rotor disc. An overview of the model formulation, as well as several verification and validation test cases are presented and discussed.
\end{abstract}

Index Terms-Contact mechanics, dynamic interface analysis, dynamic finite element model, Kane's dynamic method, LuGre friction, blade element theory, shipboard operations, simulation.

\section{INTRODUCTION}

Unmanned aircraft systems (UAS) are seeing increased use in industries ranging from environmental monitoring and protection to naval operations, and even parcel delivery. Their capacity to operate in environments hazardous to humans, their preclusion of human error during operations, and progressively-declining operating costs are some of the motivations driving the increasing use of UASs. Maritime shipboard operations in extreme weather is an ideal example of a task considered hazardous to humans for which UASs offer a widely-recognized beneficial alternative.

Currently, assistive landing, securing and handling devices exist for conventional helicopters with wheeled landing gear used in shipboard operations. Accordingly, simulation software specifically targeted at studying the dynamics of shipboard operations for these types of helicopters is available, e.g. HeliMan [1], DYNAFACE ${ }^{\circledR}$ [2], and SSMASH [3]. With UASs, a different set of challenges is present in shipboard operations.

As with helicopters, managing ship deck and aircraft dynamics for UASs is critical. However, for UASs, aerodynamic forces acting on the UAS become even more important due to the higher thrust to weight ratio of UASs compared to helicopters. Furthermore, UASs often use less compliant 'skidtype' landing gear unlike conventional maritime helicopters that use compliant suspensions and grippy tires. These typ- ical UAS characteristics constrain mechanical securing and traversing options, and present more complex ship-helicopter interface behaviors which must be characterized. Therefore a simulation package which can accurately capture the complex interface dynamics of rigid landing gear aircraft is necessary for the investigation of securing requirements and ensuring expected performance of all components in shipboard operations.

A fully-spacial simulation for the complete recovery flight and shipboard manoeuvring phases of shipboard helicopter operations named SRAMSS (Skid-equipped Rotary-wing Aircraft Manoeuvring and Securing Simulation) specifically for skid-type landing gear aircraft is presented in this paper. The simulation provides extensive analysis capabilities for the inair and on-deck ship-helicopter dynamic interface analysis, from ship deck station keeping to manoeuvring secured aircraft into the shipboard hangar.

This paper presents the 2D version of the SRAMSS simulation package which includes the methods used to combine flexible and rigid body dynamics of the aircraft, oriented dynamic collision response, and significant aerodynamic effects. To this end, it more specifically covers the integration of dynamic finite element equations into Kane's equations for dynamics in Section II, the application of the Separation Axis theorem in generating appropriately-oriented dynamic responses to collision in Section III, and the aerodynamic effects of body drag and rotor disc forces in Section IV. Lastly, simulation architecture, and test cases for the verification for proper implementation of the algorithms present in SRAMSS are discussed in Sections V and VI, respectively.

\section{DYNAMICS}

In general, the SRAMSS program simulates the dynamic interface between a skid-equipped rotary-wing aircraft and the designated landing area (DLA) aboard a ship. This involves the modelling of a rigid aircraft body, a landing gear, a ship deck, and a Rapid Securing Device (RSD) with associated deck, as shown in Figure 1. The latter is required by the fact that a secured aircraft without wheels is immobile; thus the RSD must have a mobile securing surface which provides shipboard manoeuvrability for the aircraft. 


\section{A. Governing Equations Using Kane's Method}

To generate the governing equations of motion representative of a UAS undergoing shipboard operations, Kane's formulation is used [3], [4]. It is a powerful method for generating the dynamics equations of the system, and easily manipulated into a first-order system which lends itself to numerical computation and state propagation. Kane's method states

$$
\overrightarrow{\mathbf{F}}_{r}+\overrightarrow{\mathbf{F}}_{r}^{*}=0, \quad r=1, \ldots, i
$$

where $F_{r}^{*}$ is the sum of the inertial forces and $F_{r}$ is the sum active forces, with $i$ being the number of generalized speeds required to describe the system. With vector math, the inertial and active forces for the $m$ bodies in the system are described as follows:

$$
\begin{aligned}
\overrightarrow{\mathbf{F}} & =\sum_{k=1}^{m}\left(\left[{ }_{k}^{N} V^{k}\right]^{T} \overrightarrow{\mathbf{R}}^{k}+\left[{ }_{k}^{N} W^{k}\right]^{T} \overrightarrow{\mathbf{T}}^{k}\right) \\
\overrightarrow{\mathbf{F}}^{*} & =\sum_{k=1}^{m}\left(\left[{ }_{k}^{N} V^{k}\right]^{T} \overrightarrow{\mathbf{R}}^{* k}+\left[{ }_{k}^{N} W^{k}\right]^{T} \overrightarrow{\mathbf{T}}^{* k}\right)
\end{aligned}
$$

where in a system of $m$ bodies, $\overrightarrow{\mathbf{R}}^{k}$ and $\overrightarrow{\mathbf{T}}^{k}$ are the resulting active force and active torque acting on the $k^{\text {th }}$ body, $\overrightarrow{\mathbf{R}}^{* k}$ and $\overrightarrow{\mathbf{T}}^{* k}$ are the translational and angular inertia of the $k^{\text {th }}$ body, and $V$ and $W$ are the translational and angular partial velocity matrices for the $k^{\text {th }}$ body relative to the inertial frame $N$, expressed in the $k^{\text {th }}$ body's frame.

\section{B. Dynamic Finite Element Model Integration}

The generic aircraft in the simulation is modelled as a single rigid body helicopter chassis with a skid-type landing gear modelled using a dynamic finite element (DFE) approach. DFE modelling is chosen due to its readily adaptable modelling of the aircraft's landing gear. At a glance, a DFE model physically resembles the skid landing gear which facilitates visual interpretation of the results. Moreover the skid-type landing gear is composed of interconnecting, long, slender beams which are easily captured by beam elements in a DFE model. Also, as will be shown, the DFE is integrated into Kane's equations to merge rigid and flexible body dynamics into a single system of dynamics equations. The DFE model takes the form

$$
[M] \ddot{\mathbf{q}}+[C] \dot{\mathbf{q}}+[K] \mathbf{q}=\mathbf{F}(\dot{\mathbf{q}}, \mathbf{q})
$$

as presented in Bathe [5]. The mass matrix in the DFE model is constructed as a consistent mass matrix, which captures the DFE model node's inertial behavior in all degrees of freedom. Consequently each node is treated as a discrete body by Equation 1 in Kane's method. Equation 4 can be manipulated into the forms presented in Equations 2 and 3:

$$
\begin{aligned}
\overrightarrow{\mathbf{F}} & =-\left[V_{n}\right]^{T}\left([C]\left[V_{n}\right] \overrightarrow{\mathbf{U}}+[K] \overrightarrow{\mathbf{q}}_{n}-\overrightarrow{\mathbf{F}}(\dot{\overrightarrow{\mathbf{q}}}, \overrightarrow{\mathbf{q}})\right) \\
\overrightarrow{\mathbf{F}}^{*} & =-\left[V_{n}\right]^{T}[M]\left(\left[V_{n}\right] \dot{\overrightarrow{\mathbf{U}}}+\overrightarrow{\mathbf{Z}}_{n}\right)
\end{aligned}
$$
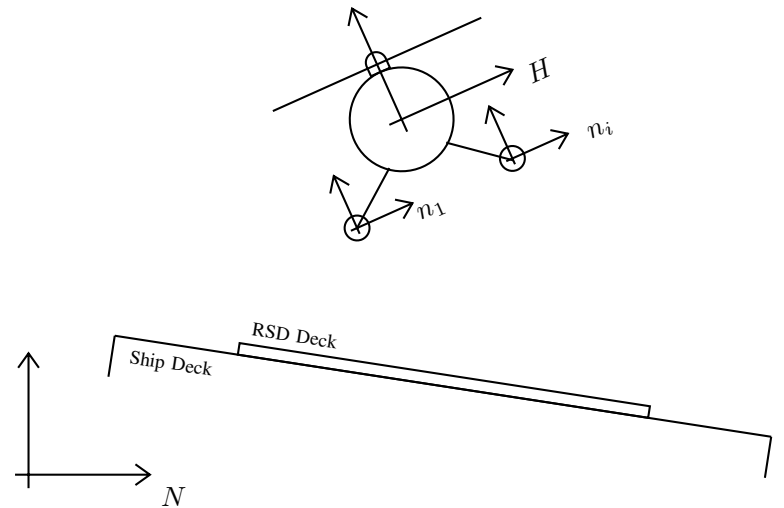

Fig. 1: SRAMSS 2D generic aircraft and ship configuration with aircraft and DFE node frames of references shown

where $[M],[C]$, and $[K]$ are the DFE consistent mass, proportional damping, and stiffness matrices, $\dot{\overrightarrow{\mathrm{U}}}$ is the vector of generalized accelerations, $\overrightarrow{\mathbf{U}}$ is the vector of generalized speeds, $\overrightarrow{\mathbf{q}}_{n}$ is the vector of generalized node positions, $\overrightarrow{\mathbf{F}}(\dot{\overrightarrow{\mathbf{q}}}, \overrightarrow{\mathbf{q}})$ is the vector of applied forces on the DFE model, and $\left[V_{n}\right]$ is the array of all translational and angular partial velocity matrices in the governing equations for a DFE model with $j$ nodes in the form

$$
\left[V_{n}\right]=\left[\begin{array}{c}
{\left[\begin{array}{c}
N \\
n_{1}
\end{array} V^{n_{1}}\right]} \\
{\left[\begin{array}{c}
N \\
n_{1}
\end{array} W^{n_{1}}\right]} \\
\vdots \\
{\left[\begin{array}{c}
N \\
n_{j}
\end{array} V^{n_{j}}\right]} \\
{\left[\begin{array}{l}
N \\
n_{j}
\end{array} W^{n_{j}}\right]}
\end{array}\right], \text { for nodes } 1, \ldots, j
$$

and $\overrightarrow{\mathbf{Z}}_{n}$ is the column array of all auxiliary terms in the governing translational and angular acceleration equations for a DFE model with $j$ nodes in the form

$$
\overrightarrow{\mathbf{Z}}_{n}=\left\{\begin{array}{c}
\vec{Z}_{n_{1}} \\
\vec{Y}_{n_{1}} \\
\vdots \\
\vec{Z}_{n_{j}} \\
\vec{Y}_{n_{j}}
\end{array}\right\} \text {, for nodes } 1, \ldots, j
$$

\section{Governing Equations For Skid-equipped Aircraft}

For the generic 2-dimensional skid-equipped aircraft shown in Figure 1, the DFE-modelled skids are attached to the rigid body of the aircraft.

The interface forces must be considered when formulating Kane's equations for both the aircraft's rigid body and the flexible DFE landing gear model. Equation 4 can be expanded to separate the external nodal forces $\mathbf{F}(\dot{\mathbf{q}}, \mathbf{q})$ into the known forces acting on the DFE nodes $\mathbf{F}_{k}$ from the unknown nodal forces acting at the nodes interfacing with the aircraft's rigid body $\mathbf{F}_{u}$, resulting in

$$
[M] \ddot{\mathbf{q}}+[C] \dot{\mathbf{q}}+[K] \mathbf{q}=\mathbf{F}_{k}(\dot{\mathbf{q}}, \mathbf{q})+\mathbf{F}_{u}(\dot{\mathbf{q}}, \mathbf{q})
$$


with Equation 9, the known node forces $\mathbf{F}_{k}$ are treated as part of the active forces applied on the DFE node 'bodies', and the unknown node forces $\mathbf{F}_{u}$ at the interface are treated as active forces on both the aircraft and DFE node bodies by application of Newton's third law. The complete generic aircraft governing equations in Kane's form can therefore be written

$$
\begin{aligned}
\overrightarrow{\mathbf{F}} & =\left[{ }_{H}^{N} V^{H}\right]^{T}\left(\overrightarrow{\mathbf{R}}^{H}-\left[T_{H \leftarrow n_{u}}\right] \dot{\overrightarrow{\mathbf{F}}}_{u}\right) \\
& +\left[{ }_{H}^{N} W^{H}\right]^{T}\left(\overrightarrow{\mathbf{T}}^{H}-\left[T_{H \leftarrow n_{u}}\right] \overrightarrow{\mathbf{T}}_{u}\right) \\
& -\left[V_{n}\right]^{T}\left([C]\left[V_{n}\right] \overrightarrow{\mathbf{U}}+[K] \overrightarrow{\mathbf{q}}_{n}\right) \\
& +\left[V_{n_{k}}\right]^{T} \overrightarrow{\mathbf{F}}_{k}+\left[V_{n_{u}}\right]^{T} \overrightarrow{\mathbf{F}}_{u}
\end{aligned}
$$

and

$$
\begin{aligned}
\overrightarrow{\mathbf{F}}^{*} & =-\left[{ }_{H}^{N} V^{H}\right]^{T} m_{H}\left(\left[{ }_{H}^{N} V^{H}\right] \dot{\overrightarrow{\mathbf{U}}}+\overrightarrow{\mathbf{Z}}_{H}\right) \\
& -\left[{ }_{H}^{N} W^{H}\right]^{T}\left(\left[{ }_{H} I^{H}\right]\left[{ }_{H}^{N} W^{H}\right] \dot{\overrightarrow{\mathbf{U}}}+\left[{ }_{H} I^{H}\right] \overrightarrow{\mathbf{Y}}_{H}\right. \\
& \left.+{ }_{H}^{N} \vec{\omega}^{H} \times\left[{ }_{H} I^{H}\right]_{H}^{N} \vec{\omega}^{H}\right) \\
& -\left[V_{n}\right]^{T}[M]\left(\left[V_{n}\right] \dot{\overrightarrow{\mathbf{U}}}+\overrightarrow{\mathbf{Z}}_{n}\right)
\end{aligned}
$$

with $\left[T_{H \leftarrow n_{u}}\right]$ transforming unknown node forces $\overrightarrow{\mathbf{F}}_{u}$ and torques $\overrightarrow{\mathbf{T}}_{u}$ to the aircraft frame of reference $H$, the aircraft mass $m_{H}$ and mass moment of inertia ${ }_{H} I^{H}$, the angular velocity of the aircraft body's centre of mass with respect to the inertial frame $N$ expressed in the aircraft frame ${ }_{H}^{N} \vec{\omega}^{H}$, and the sub-matrices $\left[V_{n_{k}}\right]$ and $\left[V_{n_{u}}\right]$ of $\left[V_{n}\right]$ separated into the known and unknown linear and angular partial velocity arrays in the form of Equation 7.

While there are unknown forces at the rigid-flexible-body interfaces, the set of governing equations remains full rank, and can be solved since the generalized accelerations at the interface are known to be zero. Equations 10 and 11 can be manipulated into the linear system

$$
[A]\left\{\begin{array}{c}
\dot{\overrightarrow{\mathbf{U}}} \\
\overrightarrow{\mathbf{F}}_{u}
\end{array}\right\}=\{\overrightarrow{\mathbf{B}}\}
$$

The solution of the system in Equation 12 is used to propagate the states forward in time. The complete solution is calculated at each time step evaluation. However only $\overrightarrow{\mathbf{U}}$ propagated from $\dot{\overrightarrow{\mathbf{U}}}$ in Equation 12's solution, and $\overrightarrow{\mathbf{U}}$ propagated to $\overrightarrow{\mathbf{q}}$ is required for the next time-step evaluation.

\section{CONTACt Mechanics}

Complex contact behavior arises between the aircraft and the ship deck due to the stiffness of the DFE landing gear. Treating the DFE model nodes as the contact points evaluated for collision response, force vectors can be generated for each contacting node, which then can be added into the known external forces vector $\mathbf{F}_{k}(\dot{\mathbf{q}}, \mathbf{q})$ in the DFE model in Equation 10 .

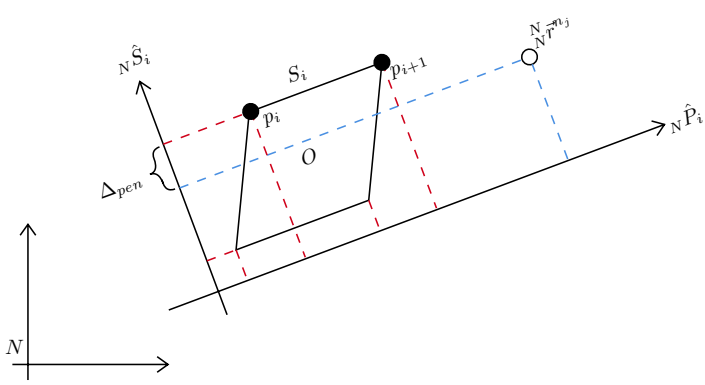

Fig. 2: Separation ${ }_{N} \hat{S}_{i}$ and parallel ${ }_{N} \hat{P}_{i}$ axes for Surface $S_{i}$ defined by points ${ }_{N}^{O} p_{i}$ and ${ }_{N}^{O} p_{i+1}$ of object $O$

\section{A. Separation Axis Theorem}

The SRAMSS program applies the Separation Axis Theorem (SAT) for collision detection [6], [7] in the contact mechanics algorithm. The SAT algorithm is chosen since it easily integrates a linear contact penalty function for penetration due to the orthogonality of the separation axes ${ }_{N} \hat{S}_{i}$ to the object $O$ surfaces $S_{i}$. Additionally, friction can be modelled by generating axes ${ }_{N} \hat{P}_{i}$ parallel to the surfaces of the object in contact as shown in Figure 2.

Computationally, the efficiency of the SAT algorithm at the core of the contact mechanics algorithm is increased in multiple ways. First, by treating the DFE nodes involved in the collision as single points to be evaluated. Second, only evaluating the DFE nodes expected to make physical contact under normal operation. Lastly, by nature of the system itself, where a limited number of simple collision objects (polygons with low separation axes counts) are defined. In the case of the 2D implementation of SRAMSS, this involves only the left and right foot nodes of the DFE landing gear model, two rectangular polygons to represent the RSD deck and ship deck, and two right trapezoids to represent the RSD securing system as shown in Figure 5.

\section{B. Oriented Dynamic Response}

To apply an appropriately-oriented response to collision, The SAT algorithm tracks which surface is contacted by the DFE model nodes. For a 2D polygon $O$ (collision object) with points ${ }_{N}^{O} p$ defined in a clockwise order, expressed in the inertial frame $N$, using left normal unit vector ${ }_{N} \hat{S}_{i}$ and parallel unit vector ${ }_{N} \hat{P}_{i}$ to surface $i$ enables calculation of contact penalty forces along $N \hat{S}_{i}$, and friction forces along ${ }_{N} \hat{P}_{i}$.

The contact penalty force for the $i^{\text {th }}$ contacted surface of object $O$ is calculated by projecting the polygon points and the $j^{\text {th }}$ DFE contact node along ${ }_{N} \hat{S}_{i}$. The contact penalty force ${ }_{\hat{S}_{i}}^{n_{j}} F^{O}$ of polygon $O$ on DFE node $n_{j}$ expressed along $\hat{S}_{i}$ is calculated by the unilateral linear penalty function 


$$
\begin{aligned}
& { }_{\hat{S}_{i}}^{n_{j}} F^{O}= \\
& \left\{\begin{array}{cl}
0 & ,{ }_{N}^{N} \overrightarrow{\mathbf{r}}^{n_{j}} \cdot{ }_{N} \hat{S}_{i} \geq \max \left({ }_{N}^{N} \overrightarrow{\mathbf{r}}^{p_{O}} \cdot{ }_{N} \hat{S}_{i}\right) \\
\begin{array}{c}
K_{\text {pen }} \Delta_{p e n} \\
+C_{\text {pen }} \dot{\Delta}_{p e n}
\end{array} & ,{ }_{N}^{N} \overrightarrow{\mathbf{r}}^{n_{j}} \cdot{ }_{N} \hat{S}_{i}<\max \left({ }_{N}^{N} \overrightarrow{\mathbf{r}}^{p_{O}} \cdot{ }_{N} \hat{S}_{i}\right)
\end{array}\right.
\end{aligned}
$$

with penalty depth $\Delta_{p e n}$ and penalty relative velocity $\dot{\Delta}_{\text {pen }}$ along $\hat{S}_{i}$, penalty stiffness coefficient $K_{p e n}$, and penalty damping coefficient $C_{\text {pen }}$.

The contact friction force for the contacted surface $S_{i}$ for object $O$ is calculated with a frictional slider model by projecting the $j^{\text {th }}$ DFE model node and associated initial object contact point (OCP) along ${ }_{N} \hat{P}_{i}$. The slider friction model in SRAMSS is an implementation of the LuGre friction model such that it can capture static and dynamic phenomena via a Stribeck function [8], [9]. The contact friction force ${ }_{P_{i}}^{n_{j}} F^{O}$ of polygon $O$ on DFE node $n_{j}$ expressed along $P_{i}$ is calculated using

$$
\begin{aligned}
& { }_{\hat{P}_{i}}^{n_{j}} F^{O}= \\
& \left\{\begin{array}{cl}
0 & ,{ }_{N}^{N} \overrightarrow{\mathbf{r}}^{n_{j}} \cdot{ }_{N} \hat{S}_{i} \geq \max \left({ }_{N}^{N} \overrightarrow{\mathbf{r}}^{p_{O}} \cdot{ }_{N} \hat{S}_{i}\right) \\
F_{L}\left({ }_{N}^{N} \overrightarrow{\mathbf{r}}^{n_{j}} \cdot{ }_{N} \hat{P}_{i}\right. & ,{ }_{N}^{N} \overrightarrow{\mathbf{r}}^{n_{j}} \cdot{ }_{N} \hat{S}_{i}<\max \left({ }_{N}^{N} \overrightarrow{\mathbf{r}}^{p_{O}} \cdot{ }_{N} \hat{S}_{i}\right) \\
\left.{ }_{N} \overrightarrow{\mathbf{r}}^{O C P_{j}} \cdot{ }_{N} \hat{P}_{i}\right) &
\end{array}\right.
\end{aligned}
$$

where $F_{L}$ is the function which models the LuGre friction model. The complete contact force on node $n_{j}$ by contacted surface $i$ on object $O$, expressed in frame $n_{j}$ is given by

$$
{ }_{n_{j}}^{n_{j}} \overrightarrow{\mathbf{F}}^{O}=\left[T_{n_{j} \leftarrow N}\right]\left(\begin{array}{l}
n_{j} \\
\hat{P}_{i}
\end{array} F_{N}^{O} \hat{P}_{i}+{ }_{\hat{S}_{i}}^{n_{j}} F^{O}{ }_{N} \hat{S}_{i}\right)
$$

where the normal penalty force and friction force scalar values are multiplied by their respective axis unit vectors which are defined in the inertial frame $N$, then transformed to the $n_{j}{ }^{\text {th }}$ node's frame as required by Equation 10. The complete contact force ${ }_{n_{j}}^{n_{j}} \overrightarrow{\mathbf{F}}^{O}$ is added to the DFE model external forces vector $\mathbf{F}_{k}$ at the appropriate element locations.

\section{AERODYNAMICS}

Inherent from the nature of the system being simulated, aerodynamic forces acting-on, or generated by the aircraft are phenomena that are necessary to be calculated. There are two major aerodynamic phenomena which must be considered. The first being the aerodynamic drag forces exerted on the aircraft body due to wind velocity relative to the aircraft's body. The second being the forces and moments acting on the aircraft body due to the uneven thrust generated by the rotor blade disc.

Additionally, due to the spin of the rotor blades there are gyroscopic effects acting on the aircraft body. However, for the 2D case they are not applicable. There are no considerations made for the influence of the ship's superstructure in the characterization of the wind's flow field within the simulation environment. Further, the orientation of the wind vector is assumed to be uniform across the ship deck.

\section{A. Aircraft Body Drag Forces}

Similar to DYNAFACE ${ }^{\circledR}$ [10] and SSMASH [3], SRAMSS models aerodynamic drag forces by using equivalent projected flat-plate areas which allows simplifications in the the aerodynamic drag equations by treating the drag coefficient as unity [11]. Thus aerodynamic drag forces on the aircraft are implemented as

$$
{ }_{H}^{H} \overrightarrow{\mathbf{F}}^{W}=\frac{1}{2} \rho \overrightarrow{\mathbf{A}}_{e q}{ }_{H}^{C P} \vec{v}^{W}\left|{ }_{H}^{C P} \vec{v}^{W}\right|
$$

where

$$
\overrightarrow{\mathbf{A}}_{e q}=\left\{\begin{array}{c}
H A_{e q, x} \\
{ }_{H} A_{e q, y} \\
H A_{e q, z}
\end{array}\right\}
$$

and ${ }_{H}^{H} \overrightarrow{\mathbf{F}}^{W}$ is the aerodynamic drag force on the aircraft body $H$, expressed in the aircraft body's frame of reference $H$, with ${ }_{H}^{C P} \vec{v}^{W}$ the wind velocity relative to the centre of pressure $C P$ of the aircraft body expressed in the aircraft frame $H$, and $\rho$ the density of the air at sea level. In Equation 16 the vectors are multiplied element-wise. The aerodynamic drag force is expressed in the $H$ frame since it must be included in the active forces vector acting on the aircraft body $\overrightarrow{\mathbf{R}}^{H}$ per Equation 10.

\section{B. Rotor Disc Thrust and Moment}

Where DYNAFACE ${ }^{\circledR}$ models rotor thrust during landing transients and induced rotor forces from free-spinning rotor blades separately [10], SRAMSS unifies these two types of forces by implementing a Blade Element Model (BEM) which can characterize the thrust forces and moments on the aircraft at any point [12]. Blade element modelling is chosen since it is infinitely configurable for any type of rotor blade and aerofoil shape, and provides accuracy to the extent desired by inclusion of different rotor blade phenomena. Additionally, it does not rely on aircraft specific thrust and moment data tables, often protected information by aircraft manufacturers.

The BEM shown in Figures 3 and 4 captures these forces by prescribing rotor blade rpm $\omega$, rotor blade physical properties (number of blades, rotor blade effective inner and outer radii, element-wise chord length $c(r)$ and blade twist $\theta_{t}(r)$ ), and blade collective $\theta_{c o l}$ and cyclical $\theta_{c y c}(\psi)$ settings.

In the BEM, each rotor blade is divided into rotor blade elements (RBEs) with width $\mathrm{d} r$. The average thrust and moment for an RBE is taken around one full revolution of the rotor disc. Then the sum of each average RBE thrust and moment is taken over the length of the rotor blade and multiplied by the number of rotor blades. First the thrust and moment for an RBE acting on the aircraft body ${ }_{H}^{H} \overrightarrow{\mathbf{F}}^{R B E}$ and ${ }_{H}^{H} \overrightarrow{\mathbf{M}}^{R B E}$ as a functions of blade azimuth $\psi$, rotor rpm, and $\mathrm{RBE}$ radius $r$ are

$$
{ }_{H}^{H} \overrightarrow{\mathbf{F}}^{R B E}(\psi, r, \omega)=\left[T_{H \leftarrow R}\right]{ }_{R}^{R} \overrightarrow{\mathbf{F}}^{R B E}(\psi, r, \omega)
$$

with 


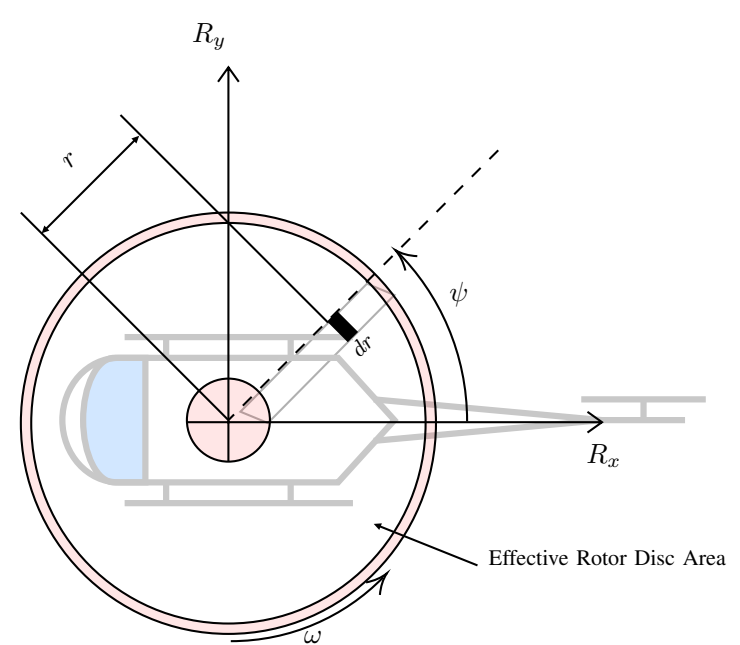

Fig. 3: Rotor Blade Element Model

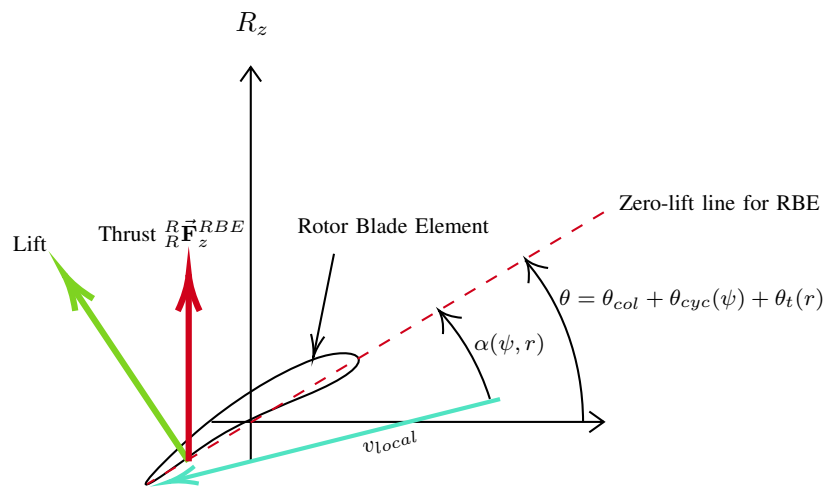

Fig. 4: Rotor Blade Element Pitch and Thurst Diagram

$$
\begin{aligned}
& { }_{R}^{R} \overrightarrow{\mathbf{F}}_{x}^{R B E}(\psi, r, \omega)=0 \\
& { }_{R}^{R} \overrightarrow{\mathbf{F}}_{y}^{R B E}(\psi, r, \omega)=0 \\
& { }_{R}^{R} \overrightarrow{\mathbf{F}}_{z}^{R B E}(\psi, r, \omega)= \\
& \quad \frac{1}{2} C_{L}(\psi, r) \rho v_{\text {local }}(\psi, r, \omega)^{2} c(r) d r \cos (\theta(\psi, r))
\end{aligned}
$$

and

$$
{ }_{H}^{H} \overrightarrow{\mathbf{M}}^{R B E}(\psi, r, \omega)={ }_{H}^{H} \overrightarrow{\mathbf{r}}^{R B E}(\psi) \times{ }_{H}^{H} \overrightarrow{\mathbf{F}}^{R B E}(\psi, r, \omega)
$$

where $C_{L}(\psi, r)$ is the coefficient of lift as a linear function of the angle of attack $\alpha(\psi, r), v_{\text {local }}(\psi)$ is the local flow velocity over the RBE tangential to the path of the RBE, $c(r)$ is the RBE chord length at radius $r, \theta(\psi, r)$ is the total pitch angle of the RBE normal to the plane of the rotor disc, and ${ }_{H}^{H} \overrightarrow{\mathbf{r}}^{R B E}$ is the position of the RBE relative to the aircraft expressed in the aircraft frame.

For a rotor disc divided into $n_{s e c}$ sectors with the $i^{\text {th }}$ disc sector having a rotor blade azimuth of $\psi_{i}$, the average thrust and moment of the $j^{\text {th }} \mathrm{RBE}$ at radius $r_{j}$ over one full revolution around the rotor disc is given by

$$
{ }_{H}^{H} \overrightarrow{\mathbf{F}}_{\text {avg }}^{R B E}\left(r_{j}, \omega\right)=\frac{1}{n_{\text {sec }}} \sum_{i=1}^{n_{\text {sec }}}{ }_{H}^{H} \overrightarrow{\mathbf{F}}^{R B E}\left(\psi_{i}, r_{j}, \omega\right)
$$

and

$$
{ }_{H}^{H} \overrightarrow{\mathbf{M}}_{\text {avg }}^{R B E}\left(r_{j}, \omega\right)=\frac{1}{n_{\text {sec }}} \sum_{i=1}^{n_{\text {sec }}}{ }_{H}^{H} \overrightarrow{\mathbf{M}}^{R B E}\left(\psi_{i}, r_{j}, \omega\right)
$$

The average thrust and torque for the $j^{\text {th }}$ RBE is summed over the length of a rotor blade composed of $n_{R B E}$ RBEs, each with radius $r_{j}$, then multiplied by the number of blades $n_{\text {blade }}$ to obtain the total thrust and moment from the rotor disc acting on the aircraft body

$$
{ }_{H}^{H} \overrightarrow{\mathbf{F}}^{R}=n_{\text {blade }} \sum_{j=1}^{n_{R B E}}{ }_{H}^{H} \overrightarrow{\mathbf{F}}_{\text {avg }}^{R B E}\left(r_{j}, \omega\right)
$$

and

$$
{ }_{H}^{H} \overrightarrow{\mathbf{M}}^{R}=n_{\text {blade }} \sum_{j=1}^{n_{R B E}}{ }_{H}^{H} \overrightarrow{\mathbf{M}}_{\text {avg }}^{R B E}\left(r_{j}, \omega\right)
$$

The BEM model implemented in SRAMSS currently does not consider any aerodynamic phenomena related to the inner or outer tips of the rotor blades, drag divergence, or ground effects.

\section{Simulation Architecture}

SRAMSS is a state propagation program which has been written in the interpreted language MATLAB ${ }^{\circledR}$ for ease of verification, then ported to the compiled language FORTRAN90 to increase performance for more computationally-intensive simulations.

It solves the system presented in Equation 12 and propagates the generalized accelerations vector $\dot{\vec{U}}$ and generalized speeds vector $\overrightarrow{\mathbf{U}}$ to the next time step using an ordinary differential equation (ODE) solver. The solver selected is suited to stiff systems as is required by the implementation of the DFE model landing gear, and has function root-finding capabilities. The root-finding capabilities are leveraged to terminate integration steps at time $t_{\text {contact }}$ where the DFE model landing gear nodes come into, or are released from, contact with the deck, as this results in a change in system dynamics at $t_{\text {contact }}$. The solver uses automatic selection between combinations of stiff and non-stiff multi-step implicit solvers. SRAMSS is a multi-step solver with a base output time-step increment, but maintains variable time-step output due to time-step termination and re-initialization at $t_{\text {contact }}$ times.

\section{VERIFICATION AND VALIDATION}

While the 2D version of SRAMSS discussed here cannot capture out-of-plane effects of a complete 3D simulation, it can be used to verify planar test cases for the SRAMSS equation formulations and algorithms. The generic aircraft used for verification purposes is as shown in Figure 5. In addition 


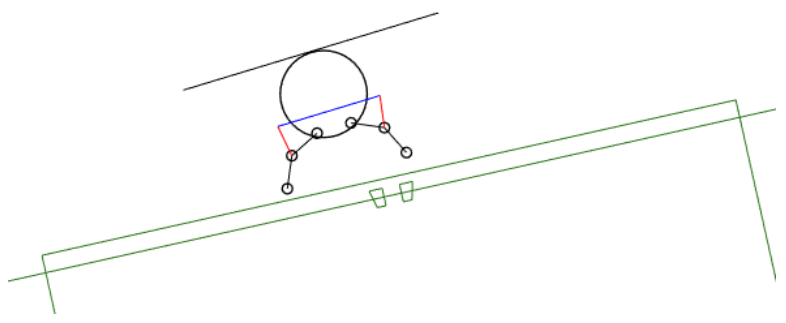

Fig. 5: MATLAB ${ }^{\circledR}$ Generic Aircraft With Discrete Landing Gear Dampers

TABLE I: Generic Aircraft Simulation Parameters

\begin{tabular}{lc}
\hline Parameter & Value \\
\hline Helicopter Properties & $50 \mathrm{~kg}$ \\
Body mass & $6.25 \mathrm{~kg} \cdot \mathrm{m}^{2}$ \\
Body Mass Moment of Inertia & $2.5 \mathrm{X} 10^{4} \mathrm{~N} / \mathrm{m}$ \\
Discrete Damper Spring Coefficient & $500 \mathrm{Ns} / \mathrm{m}$ \\
Discrete Damper Damping Coefficient & 4 \\
Number of Rotor Blades & $0.15 \mathrm{~m}$ \\
Inner Effective Rotor Disc Radius & $0.675 \mathrm{~m}$ \\
Outer Effective Rotor Disc Radius & $0 \mathrm{rad}$ \\
Rotor Blade Twist (constant) & $0.15 \mathrm{~m}$ \\
Rotor Blade Chord Length (constant) & \\
\hline Finite Element Landing Gear Properties & $6.9 X 10^{10} \mathrm{~Pa}$ \\
Element Young's Modulus & $5.00 X 10-7 \mathrm{~m}$ \\
Element Second Moment of Area & $2710 \mathrm{~kg} / \mathrm{m}^{3}$ \\
Element Density & $1.2 X 10^{-4} \mathrm{~m}{ }^{2}$ \\
Element Cross Section & $6.5 X 10^{5} \mathrm{rad} / \mathrm{s}$ \\
Rayleigh Damping Upper Frequency & 0.15 \\
Rayleigh Damping Upper Damping Ratio & $0 \mathrm{rad} / \mathrm{s}$ \\
Rayleigh Damping Lower Frequency & 0.15 \\
Rayleigh Damping Lower Damping Ratio & 6 \\
Number of Nodes & 4 \\
Number of Elements & $600 \mathrm{Ns} / \mathrm{m}$ \\
\hline Collision Mechanics Properties & $0.001 \mathrm{~m} / \mathrm{s}$ \\
Penalty Stiffness & 0.4 \\
Penalty Damping & 0.9 \\
Friction Micro-displacement Stiffness & $3130 \mathrm{Ns} / \mathrm{m}$ \\
Friction Micro-displacement Damping & $3 \times 10^{3} \mathrm{~N} / \mathrm{m}$ \\
Stribeck Velocity Threshold $v_{s}$ & \\
Dynamic Friction Coefficient $\mu_{d}$ & \\
Static Friction Coefficient $\mu_{s}$ & \\
\hline & \\
\hline
\end{tabular}

to a rigid body and two hinged DFE landing gear legs, the generic aircraft has two discrete external dampers attached between the middle nodes of the DFE landing gear legs and the aircraft body's winglets. Aircraft and contact-mechanicsrelated constant properties are listed in Table I.

The test cases presented in Table II were conducted to verify that SRAMSS captures different physical phenomena, and produces results for aircraft behavior that match predictions under given conditions.

\section{A. Case 1: Free-fall and Steady-State Posture}

The first test case is a level drop of the generic aircraft towards the RSD deck with zero-velocity initial conditions. Figure 6 shows the aircraft's body's centre of gravity $(\mathrm{CoG})$ position as the solid blue line, acceleration as the solid black line, and gravitational acceleration as the dotted line. While in the air, the aircraft maintains a downwards acceleration of
$9.81 \mathrm{~m} / \mathrm{s}^{2}$. When the aircraft's CoG falls below 0.5500 metres (in contact with RSD deck) the acceleration value deviates from $9.81 \mathrm{~m} / \mathrm{s}^{2}$. At steady-state, the acceleration falls to $0 \mathrm{~m} / \mathrm{s}^{2}$. This test verifies the correct implementation of gravity.

The root-finding function implemented into the ODE solver is also verified to be implemented correctly as the deviations in acceleration occur exactly at times where contact and release from contact occur.

Once steady state is achieved as the aircraft rests on the RSD deck, as shown in Table IV, the increase in distances of the landing gear feet (in the $\mathrm{x}$ direction) from undeformed to steady-state confirm an outward-splayed stance of the landing gear. The resting height of the $\mathrm{CoG}$ at steady-state also decreased which is expected from a splayed landing gear deforming under the weight of the aircraft.

\section{B. Case 2: Friction sliding angle}

As part of the oriented dynamic response to contact, a friction force is applied to the landing gear nodes as they slide across the contacted object. The flexibility of the contact mechanics algorithm in SRAMSS allows many friction models to be used. Currently two friction models have been implemented. The first model is a simple spring-damper frictional slider model which saturates to the Coulomb friction value. The second model is a LuGre frictional slider model which captures static friction, Coulomb (dynamic) friction, viscous friction, and pre-sliding micro displacements.

The Second test case evaluates these models through the relative sliding velocity across the RSD deck as the ship roll angle increases. For this case, friction parameters have been modified to capture the desired phenomena before the aircraft's tipping point is reached. The modified parameters of Table I can be found in Table III.

By the equation for the coefficient of friction $\mu=\tan (\theta)$ with $\theta$ being the ship's roll angle (radians), the predicted roll angle at which the aircraft should start sliding can be calculated. The aircraft should slip at $\theta=-0.1974$ radians with the spring-damper friction model using $\mu_{d}$, and at $\theta=$ -0.3806 radians with the LuGre friction model with $\mu_{s}$, given that static friction is modelled here. In Figure 7, the predictions made by theory are verified. The velocities for both models begin to increase at their respective predicted ship roll angles.

Additionally, as the ship rolls, more weight is transferred to the leading landing gear foot (right) which decreases the friction forces on the trailing foot, eventually causing it to stutter across the ship deck. Resulting oscillations in the aircraft body cause the leading landing gear foot to slightly stutter which leads to its eventual sliding motion as the spikes show in Figure 7, for $v$ LuGre around the 25 second mark.

Figure 8 presents the friction forces $F_{L u G r e}$ generated by the LuGre model on the leading landing gear foot. As the ship roll angle increases, the model settles into the range limited by the static and dynamic friction values. Where $F_{L u G r e}$ lies within that range is prescribed by the Stribeck equation

$$
F=F_{d}+\left(F_{s}-F_{d}\right) \exp \left(-\left(v / v_{s}\right)^{2}\right)
$$


TABLE II: SRAMSS 2D Verification Test Cases

\begin{tabular}{|c|c|c|c|}
\hline Case & Configuration Schematic & Purpose & Results \\
\hline $\begin{array}{l}\text { 1. Free-fall and } \\
\text { Steady-state } \\
\text { Posture }\end{array}$ & & $\begin{array}{l}\text { - Verify gravitational acceleration and } \\
\text { numerical integration implemented } \\
\text { correctly. } \\
\text { - Deformed steady-state posture on deck } \\
\text { (Splayed landing gear and aircraft CoG } \\
\text { closer to deck. }\end{array}$ & $\begin{array}{l}\text { - Refer to Figure } 6 \text { in Appendix A for } \\
\text { acceleration plot. } \\
\text { - Refer to Table IV in Appendix A for } \\
\text { deformed aircraft values. }\end{array}$ \\
\hline $\begin{array}{l}\text { 2. Friction } \\
\text { sliding angle }\end{array}$ & & $\begin{array}{l}\text { - Compare behavior of LuGre and simple } \\
\text { spring-damper frictional slider models. } \\
\text { - Verify that the LuGre friction model } \\
\text { (which models static friction) lets the } \\
\text { aircraft slip at a larger ship roll angle. }\end{array}$ & $\begin{array}{l}\text { - Figure } 7 \text { in Appendix A shows sliding } \\
\text { velocities over time against ship roll } \\
\text { angle for the LuGre and spring-damper } \\
\text { frictional slider models. } \\
\text { - Figure } 8 \text { in Appendix A shows the } \\
\text { generated friction forces for the LuGre } \\
\text { model against the roll angle of the ship } \\
\text { over time. }\end{array}$ \\
\hline $\begin{array}{l}3.1 \text { Uniform } \\
\text { Rotor Disc } \\
\text { Thrust }\end{array}$ & & $\begin{array}{l}\text { - Verify that the BEM is implemented } \\
\text { correctly. } \\
\text { - Verify aircraft will generate uniform } \\
\text { thrust in zero wind conditions. }\end{array}$ & $\begin{array}{l}\text { - Figure } 9 \text { in Appendix A shows } \\
\text { displacement, thrust and moment on } \\
\text { aircraft body plots. }\end{array}$ \\
\hline $\begin{array}{l}3.2 \text { Rotor Disc } \\
\text { Thrust with } \\
\text { Relative Wind }\end{array}$ & Headwind & $\begin{array}{l}\text { - Continuation of Case } 3.1 \text { for BEM } \\
\text { verification. } \\
\text { - Verify non-uniform thrust and moment } \\
\text { generation in headwinds. }\end{array}$ & $\begin{array}{l}\text { - Figure } 10 \text { in Appendix A shows } \\
\text { displacement, thrust and moment on } \\
\text { aircraft body plots. }\end{array}$ \\
\hline $\begin{array}{l}3.3 \text { Rotor Disc } \\
\text { Thrust With } \\
\text { Relative Wind } \\
\text { and Cyclic } \\
\text { Compensation }\end{array}$ & Headwind & $\begin{array}{l}\text { - Continuation of Case } 3.1 \text { and } 3.2 \text { for } \\
\text { BEM verification. } \\
\text { - Verify cyclic setting compensation on } \\
\text { retreating blades side generates enough } \\
\text { thrust to counter pitching (roll) moment } \\
\text { on aircraft. }\end{array}$ & $\begin{array}{l}\text { - Figure } 11 \text { in Appendix A shows } \\
\text { displacement, thrust and moment on } \\
\text { aircraft body plots. }\end{array}$ \\
\hline $\begin{array}{l}\text { 4. RSD } \\
\text { Securing } \\
\text { Concept }\end{array}$ & & $\begin{array}{l}\text { - Verify Implementation of RSD deck } \\
\text { securing device. } \\
\text { - Verify Aircraft's response to securing } \\
\text { mechanism. }\end{array}$ & $\begin{array}{l}\text { - Figure } 12 \text { in Appendix A shows the } \\
\text { undeformed, and secured posture of the } \\
\text { aircraft. }\end{array}$ \\
\hline $\begin{array}{l}5 . \\
\text { Comprehensive } \\
\text { Phenomena } \\
\text { Test }\end{array}$ & $\leftarrow$ & $\begin{array}{l}\text { - Verify aircraft behavior under } \\
\text { fully-dynamic conditions. } \\
\text { - RSD deck and aircraft have non-zero } \\
\text { initial velocity conditions. } \\
\text { - Verify collision behavior with multiple } \\
\text { objects (RSD and ship decks). } \\
\text { - Verify securing mechanism of RSD } \\
\text { deck. }\end{array}$ & $\begin{array}{l}\text { - Figure } 13 \text { provides transient settings for } \\
\text { the rotor disc and wind conditions. } \\
\text { - Figure } 14 \text { in Appendix A shows } \\
\text { snapshots of test Case } 5 \text { at significant } \\
\text { timestamps. }\end{array}$ \\
\hline
\end{tabular}

TABLE III: Modified Aicraft Simulation Parameters

\begin{tabular}{lc} 
Parameter & Value \\
\hline Collision Mechanics Properties & \\
Friction Micro-displacement Stiffness & $5 \times 10^{5} \mathrm{~N} / \mathrm{m}$ \\
Friction Micro-displacement Damping & $5 \times 10^{4} \mathrm{Ns} / \mathrm{m}$ \\
Stribeck Velocity Threshold $v_{s}$ & $0.01 \mathrm{~m} / \mathrm{s}$ \\
Dynamic Friction Coefficient $\mu_{d}$ & 0.2 \\
Static Friction Coefficient $\mu_{s}$ & 0.4 \\
\hline
\end{tabular}

However, the forces do not immediately fall within the range since the frictional slider portion of the LuGre model requires displacement in the aircraft landing gear to generate friction forces, whereas the normal forces required for the friction force range are immediately present at contact. As the ship roll angle increases, the Friction force approaches the upper limit of static friction $F_{s}$ since the sliding velocity $v$ is still small. Around 26 seconds, $F_{L u G r e}$ saturates to this limit which allows the aircraft to begin sliding. With the increase in $v$ exceeding the Stribeck velocity threshold $v_{s}, F_{\text {LuGre }}$ approaches $F_{d}$ by Equation 25. Spikes in $F_{L u G r e}, F_{s}$ and $F_{d}$ are caused by the transfer of weight between the two landing gear feet which allows for the trailing foot to stutter across the ship deck intermittently.

The parameters used for this test case are arbitrary, and tuned to visually show the desired phenomena. True parameters will be determined through extensive experimental testing. Nevertheless, The LuGre model is verified to capture the friction phenomena predicted.

\section{Case 3.1: Uniform Rotor Disc Thrust}

Test Cases 3.1 through 3.3 verify the implementation of the rotor blade element model. Test case 3.1 verifies the uniform generation of thrust by the rotor disc without relative wind on deck, and a constant collective blade pitch setting 
of $\theta_{\text {collective }}=0.14 \mathrm{rad}$. Figure 9 shows that as rotor disc RPM increases to $140 \mathrm{rad} / \mathrm{s}$, thrust generated increases towards 600 N. Since there is no relative wind, the advancing and retreating sides of the rotor disc generate equal amounts of thrust, hence the $0 \mathrm{Nm}$, which results in a vertical ascent by the aircraft. As the RPM decreases to $115 \mathrm{rad} / \mathrm{s}$, the aircraft tops out at an altitude of 1.82 metres. With the decrease in RPM, and subsequently decrease in thrust, the aircraft lands on the RSD deck. Spikes in thrust between 8 and 12 seconds are a result of relative axial airflow as the aircraft falls through the air. Strictly vertical aircraft motion, and zero rotor disc moment generation verifies uniformity in thrust generated.

\section{Case 3.2: Rotor Disc Thrust With Relative Wind}

This test case builds on Case 3.1 with the addition of a relative headwind increasing to $1 \mathrm{~m} / \mathrm{s}$, while maintaining a constant collective pitch setting $\theta_{\text {collective }}=0.14 \mathrm{rad}$. Due to the headwind, advancing rotor blades see an increase in thrust generation while the retreating blades see a loss in thrust generation. This imbalance causes a moment on the aircraft as shown in Figure 10. As the aircraft touches-off the RSD deck the moment causes the aircraft to begin to roll. The resulting change in thrust vector orientation due to roll causes the aircraft to move laterally before touching down as the rotor disc RPM decreases.

\section{E. Case 3.3: Rotor Disc Thrust With Relative Wind and Cyclic Setting Compensation}

The swashplate of the rotor assembly in a rotary wing aircraft can prescribe a harmonic change in blade pitch angle, beyond the collective setting, called the cyclic pitch setting $\theta_{c y c}$. The cyclic can be prescribed by

$$
\theta_{c y c}=\theta_{c y c, \max } \cos \left(\psi-\psi_{\max }\right)
$$

where $\theta_{c y c, \max }$ is the maximum cyclic angle occurring at rotor blade azimuth $\psi_{\max }$, with respect to the definition of the blade azimuth in Figure 3 in Section IV-B [12]. For test Case 3.3, as shown in Figure 11, there is an added cyclic input which counters the roll of the aircraft. On the advancing blades, the total pitch is reduced by $\theta_{c y c}=-0.01$ radians at $\psi=\pi / 2$. On the retreating blades, the total pitch is increased by $\theta_{c y c}=0.01$ radians at $\psi=-\pi / 2$. This cyclic setting causes the retreating blades to generate more lift than the advancing blades. The moment generated by the imbalance of thrust corrects the roll of the aircraft. This allows the aircraft to gain altitude while maintaining the lateral momentum from the initial roll. Consequently the aircraft is in flight for a for a longer duration than Case 3.2, which results in an increased lateral displacement approaching 3 metres, compared to 0.5 metres from Case 3.2.

Provided data concerning rotor blade geometry, RPM, collective and cyclic settings, the BEM model is verified to be a suitable model to calculate the thrust and pitching moment acting on the aircraft for any given rotor blade configuration. Cases 3.1-3.3 demonstrate expected aircraft behavior.

\section{F. Case 4: RSD Securing Concept}

As stated in the introduction, a secured aircraft with skidtype landing gear is immobile, thus on-deck manoeuvres require the securing device to be mobile. Consequently the RSD deck must have planar degrees of freedom. The lightweight characteristic of many UASs coupled with their limited payload capacity suggests that the securing mechanism be contained on the mobile RSD deck.

As a concept, expanding wedges which drive outwards over the skid-tubes of the landing gear provide a simple solution to securing the aircraft. The aircraft responds as expected by lowering its posture as the landing gear is splayed. Verification of the wedges as a securing solution is better showcased and verified in Case 5.

\section{G. Case 5: Comprehensive Phenomena Test}

Case 5 combines all the phenomena which SRAMSS is able to capture. As shown in Figure 14, this includes transient headwinds, a gusting crosswind, rotor disc RPM, and collective and cyclic pitch setting. Moreover sinusoidal ship heave and roll motions are applied, and once the aircraft is secured, the RSD deck performs a lateral manoeuvre of the secured aircraft.

Figure 13 showcases eventful timestamps of the test case simulation. The aircraft moves laterally towards the RSD deck aided by drag forces from the crosswinds. The cyclic input prevents the aircraft from rolling in the headwind. At 0.48 seconds the aircraft touches-off the deck, then reduces the collective and cyclic settings to 0 radians between 0.80 and 1.00 seconds. This causes the aircraft to completely touch-down at 1.19 seconds. Crosswinds prevent the aircraft from sliding over the securing wedges. Between 3.00 and 3.50 seconds the wedges are deployed to centre and secure the aircraft. Near the lowest point in the ship's heave at 7.86 seconds, the RSD deck begins the lateral manoeuvre which terminates at 10.00 seconds.

The complete test case verifies that SRAMSS can handle the complete set of phenomena implemented. Moreover it verifies the wedges as a securing mechanism for skid-type landing gear aboard ships.

\section{H. Other Remarks}

Throughout all test cases, the observation is made that the aircraft remains intact. This verifies that the combination of the rigid and flexible-body dynamics into Equations 10 and 11 of Section II are implemented correctly. This is to say that the landing gear remain attached to the aircraft body without any unexpected behavior, and hinge around their mounting points, to the extent allowed by the discrete dampers, as prescribed.

The contact mechanics algorithm which implements the equations of Section III are never explicitly the target in any test case; however each test case relies on the proper functioning of them. Case 1 verifies the impenetrability of defined polygons. Moreover, the proper implementation of gravity in conjunction with the aircraft coming to rest indicates that normal forces generated by the collision penalty function from Equation 13 are implemented correctly. Case 2 verifies 
the oriented dynamic response of the algorithm through the proper implementation of friction, which also means normal force generation is inherently implemented correctly. Test Case 6 verifies that SRAMSS applies the oriented dynamic response appropriately to multiple objects concurrently (RSD deck, 2 wedges), all while the objects are prescribed non-zero motion.

The Cases 3.1 through 3.3 verify the correct implementation of BEM theory presented in Section IV, and show that it is able to generate the appropriate rotor disc thrust and moment vectors based on aerodynamic conditions surrounding the aircraft.

\section{CONCLUSION}

Through the test cases presented in Section VI, the algorithms implemented in the 2-dimensional version of SRAMSS have been verified to function as predicted. This includes the proper implementation of gravitational, normal, frictional, drag-induced, and rotor disc forces exerted on the complete aircraft. Moreover, collision detection and response, and geometric fidelity of the DFE model under load behave as expected.

While currently in the 2D verification and validation phase, the governing equations and algorithms contained within the SRAMSS simulation package are written to be easily extended to a three-dimensional simulation environment. For example, the 3D version will have the current Euler angles used for rotation switched to Euler parameters to avoid singularities, surface normal axes for collision detection will be calculated using the unit vectors normal to the planes of the surfaces defining an object, and the DFE model will use 12-DoF beam elements. Moreover, the aerodynamics presented in Section IV are currently derived in their 3D form.

The SRAMSS simulation package has the potential to provide a comprehensive simulation environment for skidequipped rotary-wing aircraft undergoing shipboard manoeuvres. These capabilities are ultimately necessary in the study of shipboard UAS operations due to their emerging role in maritime shipboard environments.

\section{REFERENCES}

[1] D. R. Linn and R. G. Langlois, "Development and experimental validation of a shipboard helicopter on-deck maneuvering simulation," Journal of Aircraft, vol. 43, no. 4, pp. 895-906, 2006.

[2] R. G. Langlois, M. LaRosa, and D. A. R. Tadros, "Development, validation, and application of the dynaface ${ }^{\circledR}$ helicopter/ship dynamic interface simulation software package," vol. 35, San Diego, CA, 2003 , edited by V. W. Ingalls.

[3] M. J. Léveillé and R. G. Langlois, "Spacial modelling and simulation of on-deck helicopter securing and manoeuvring," in Proceedings of the 70th Annual Forum of the American Helicopter Society, Montreal, QC, Canada, May 2014.

[4] A. Purushotham and J. Anjeneyulu, "Kane's method for robotic arm dynamics: a novel approach," IOSR Journal of Mechanical and Civil Engineering (IOSR-JMCE), vol. 6, no. 4, pp. 7-13, May-June 2013.

[5] K.-J. Bathe, Finite Element Procedures. New Jersey: Prentice Hall, 1996, p. 768.

[6] C. Liang and X. Liu, "The research of collision detection algorithm based on separating axis theorem," International Journal of Science, vol. 2, no. 10, pp. 110-114, 2015.

[7] S. Gottschalk, "Separating axis theorem," Department of Computer Science, UNC Chapel Hill, Tech. Rep. TR96-024, 1996.
[8] C. C. de Wit, H. Olsson, K. J. Astrom, and P. Lishinsky, "A new model for control of systems with friction," IEEE Transactions On Automatic Controls, vol. 40, no. 3, pp. 418-425, March 1995.

[9] Y. Liu, J. Li, Z. Zhang, X. Hu, and W. Zhang, "Experimental comparison of five friction models on the same test-bed of the micro stick-slip motion system," Mechanical Sciences, vol. 6, pp. 15-28, 032015.

[10] D. R. G. Langlois and D. A. R. Tadros, "User's manual for the aircraft/ship dynamic interface simulation dynaface ${ }^{\circledR}$ release 5.0.” Indal Technologies Inc, Mississauga, ON, Canada, Tech. Rep. ITI Report No. 99/419, January 1998.

[11] B. W. McCormick, Aerodynamics, Aeronautics, and Flight Mechanics. Wiley, August 1979, p. 196.

[12] W. Z. Stepniewski, "Basic theories of rotor aerodynamics (with application to helicopters)," Boeing Vertol Company, Philadelphia, PA, Tech. Rep. N79-22039, January 1979.

\section{APPENDIX}

\section{A. Test Cases Figures}

TABLE IV: (Case 1) Undeformed vs Steady-state Helicopter Posture

\begin{tabular}{lrr}
\hline DoF & Underformed [m] & Steady-state [m] \\
\hline aircraft CoG x & 0.0000 & 0.0000 \\
aircraft CoG y & 0.4500 & 0.4345 \\
Left Foot x & -0.3500 & -0.3652 \\
Left Foot y & 0.0000 & 0.0000 \\
Right Foot x & 0.3500 & 0.3652 \\
Right Foot y & 0.0000 & 0.0000 \\
\hline
\end{tabular}

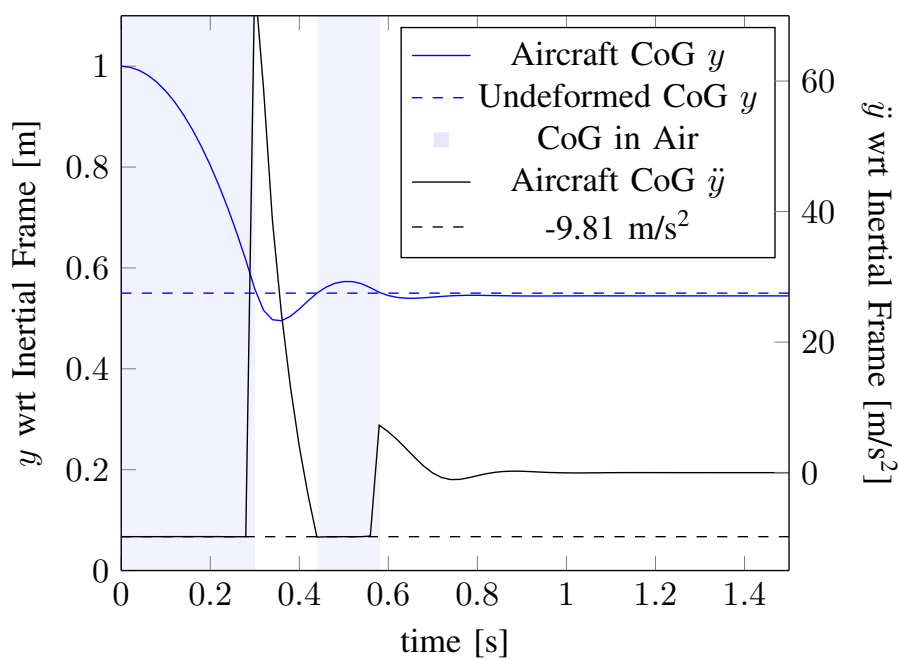

Fig. 6: (Case 1) Aircraft CoG y-position and y-acceleration with respect to the inertial frame. The dashed line is the height of the aircraft body $\mathrm{CoG}$ resting on a level 0.1 metres thick RSD deck with respect to the inertial frame, with an undeformed landing-gear 


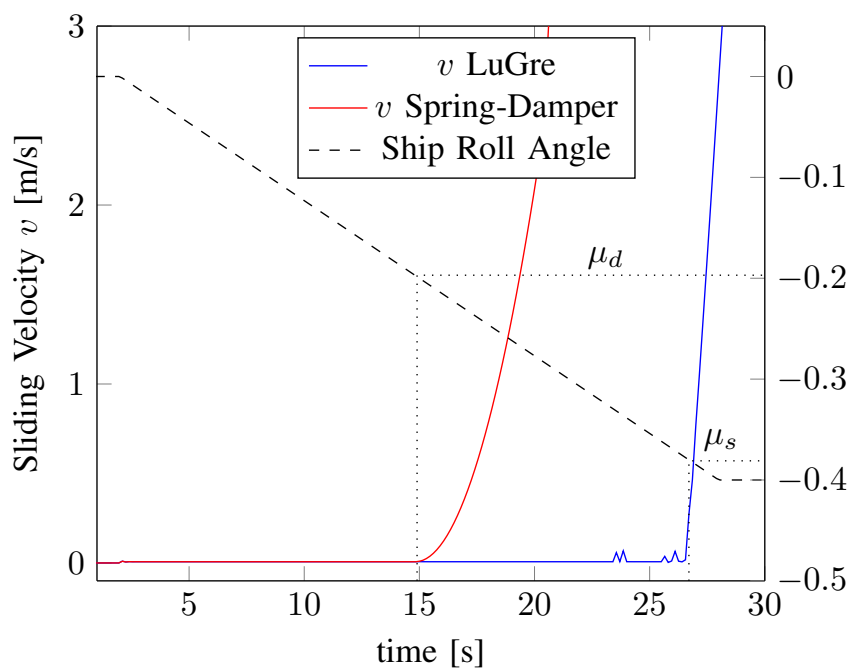

Fig. 7: (Case 2) Sliding velocity of leading (right) landing gear foot along the RSD deck as the roll angle of the ship increases

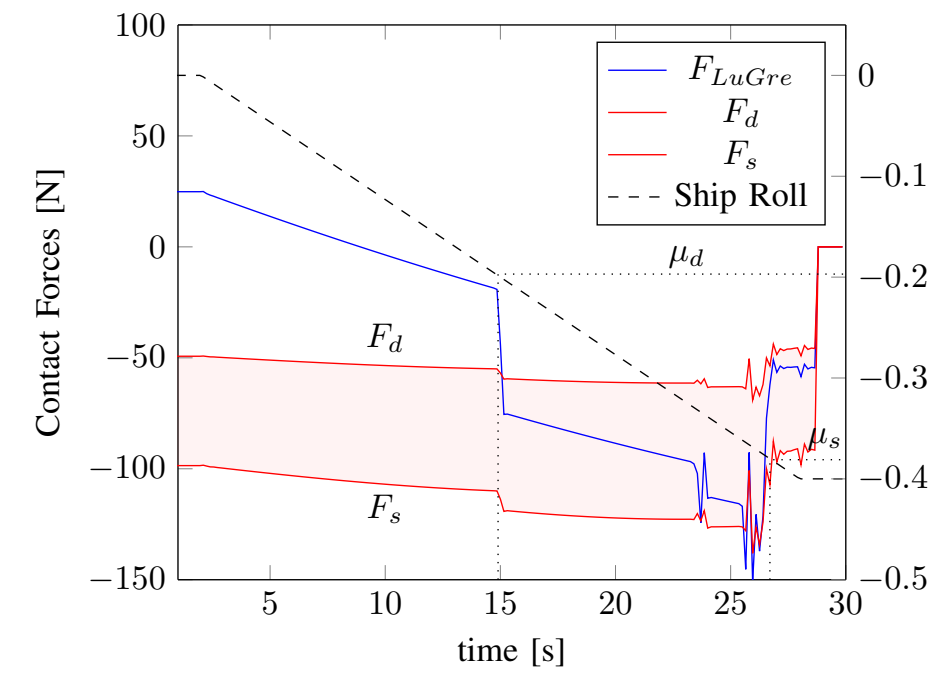

Fig. 8: (Case 2) Friction forces of leading (right) landing gear foot along the RSD deck as the roll angle of the ship increases

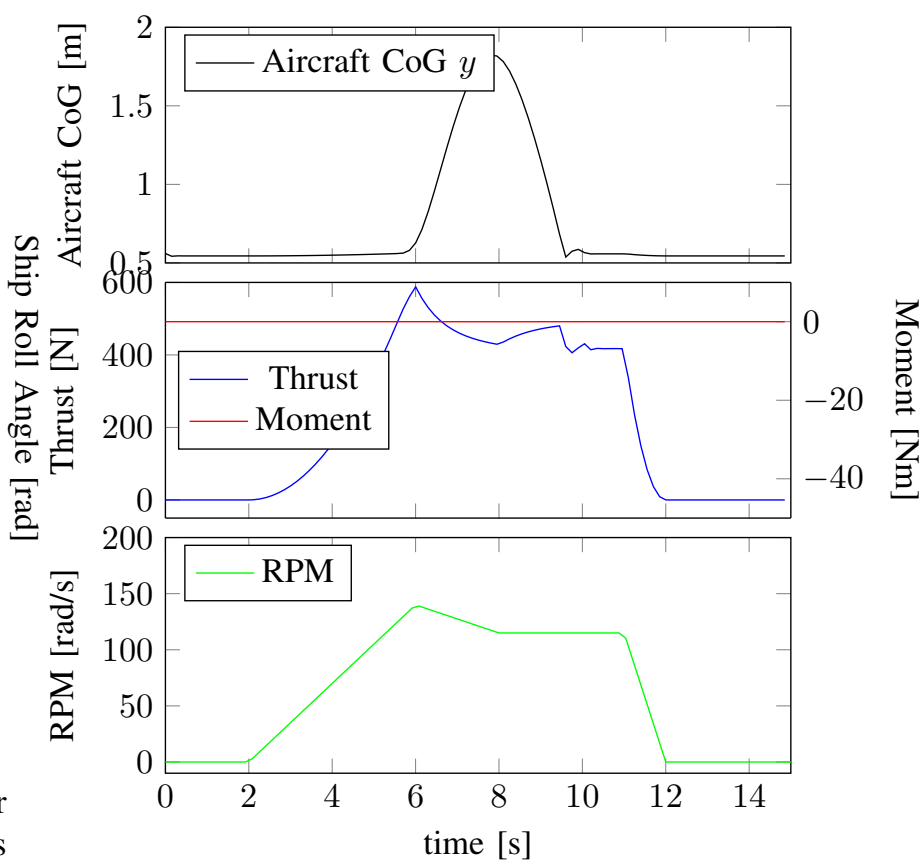

Fig. 9: (Case 3.1) Uniform rotor disc thrust with no crosswinds

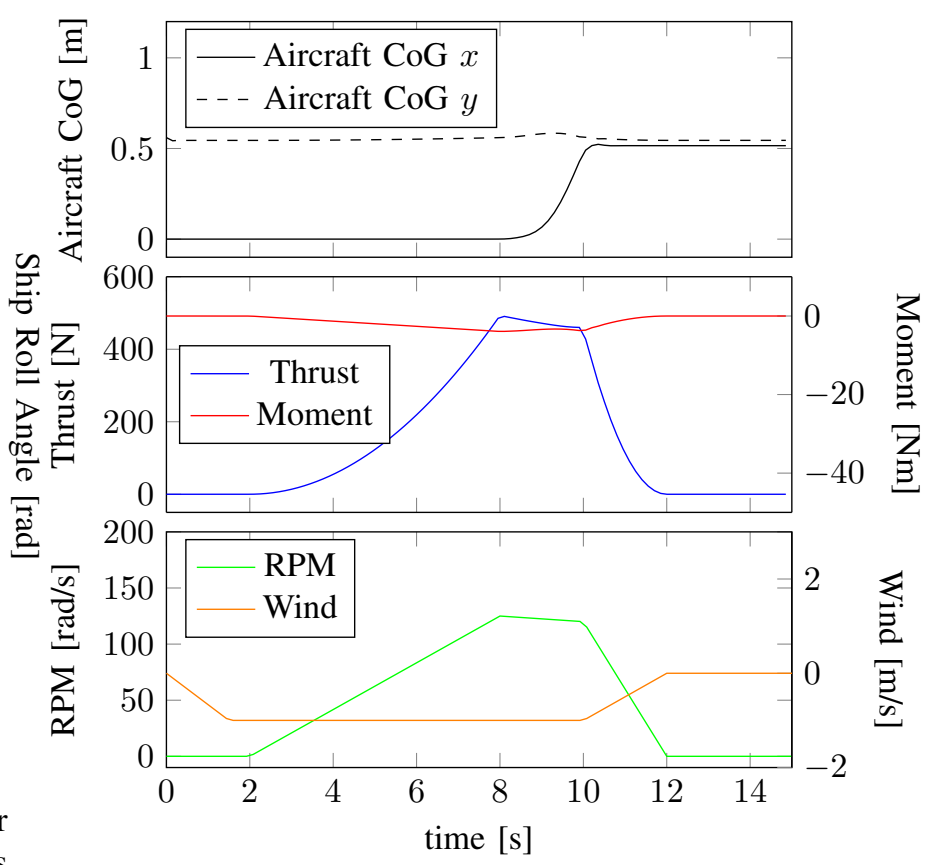

Fig. 10: (Case 3.2) Rotor disc forces and aircraft displacement in headwind 


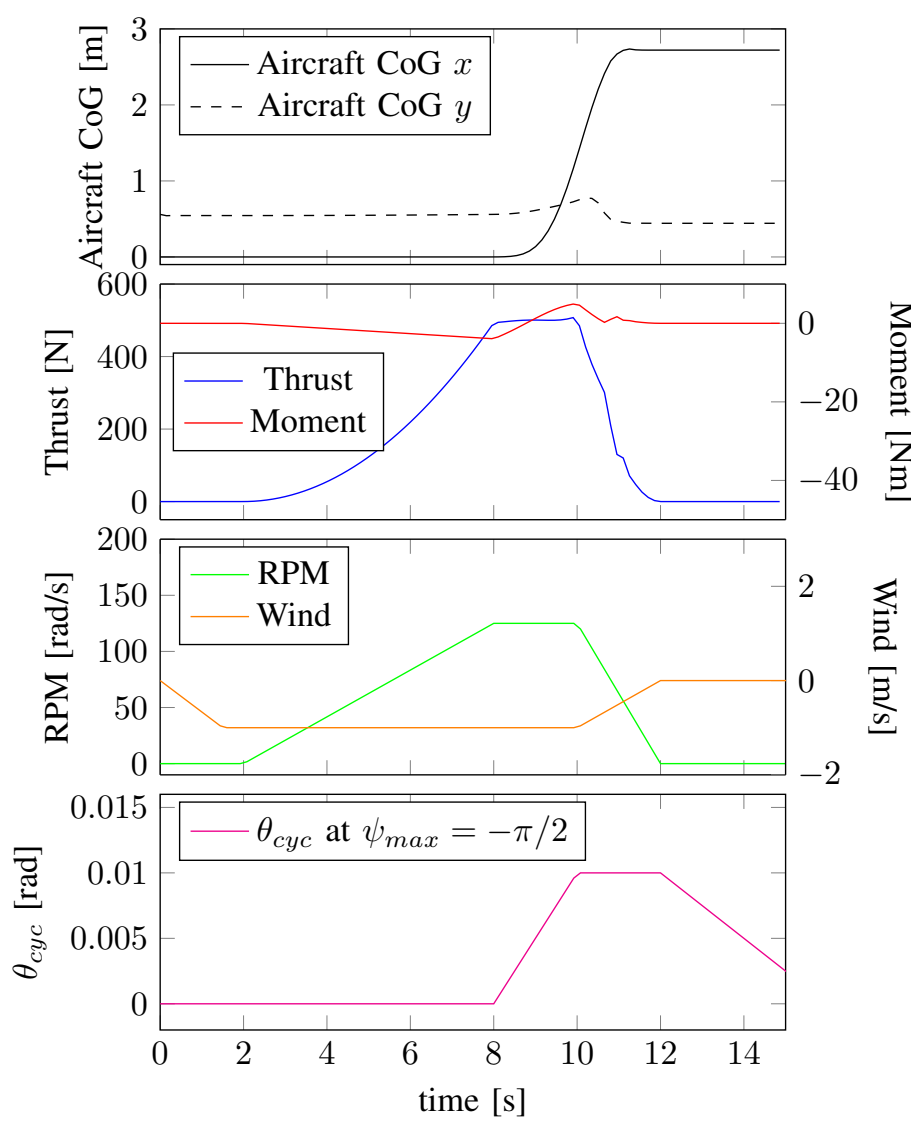

Fig. 11: (Case 3.3) Rotor disc forces and aircraft displacement in headwind, with cyclic pitch compensation

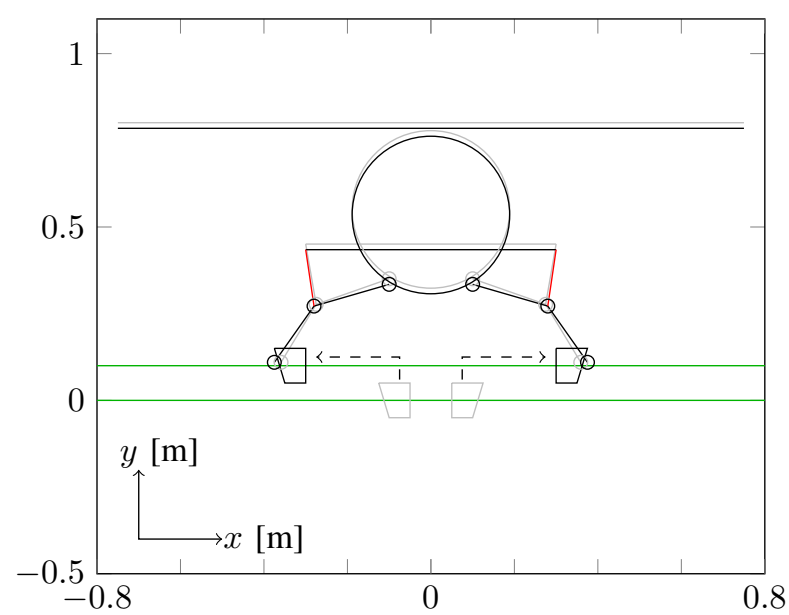

Fig. 12: (Case 4) RSD Deck securing wedge concept. Light gray outline is aircraft initial posture, black outline is aircraft posture after securing.
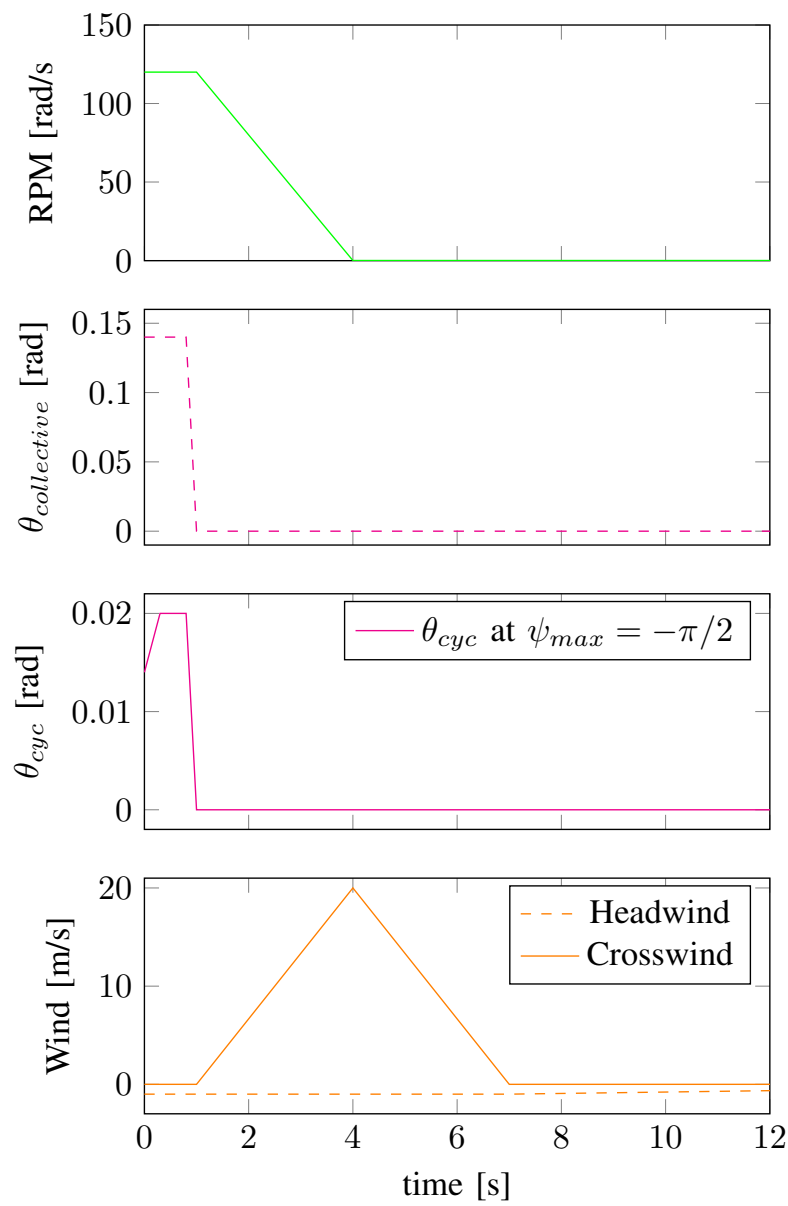

Fig. 13: (Case 5) Transient aerodynamic effects: wind speed, rotor disc parameters 

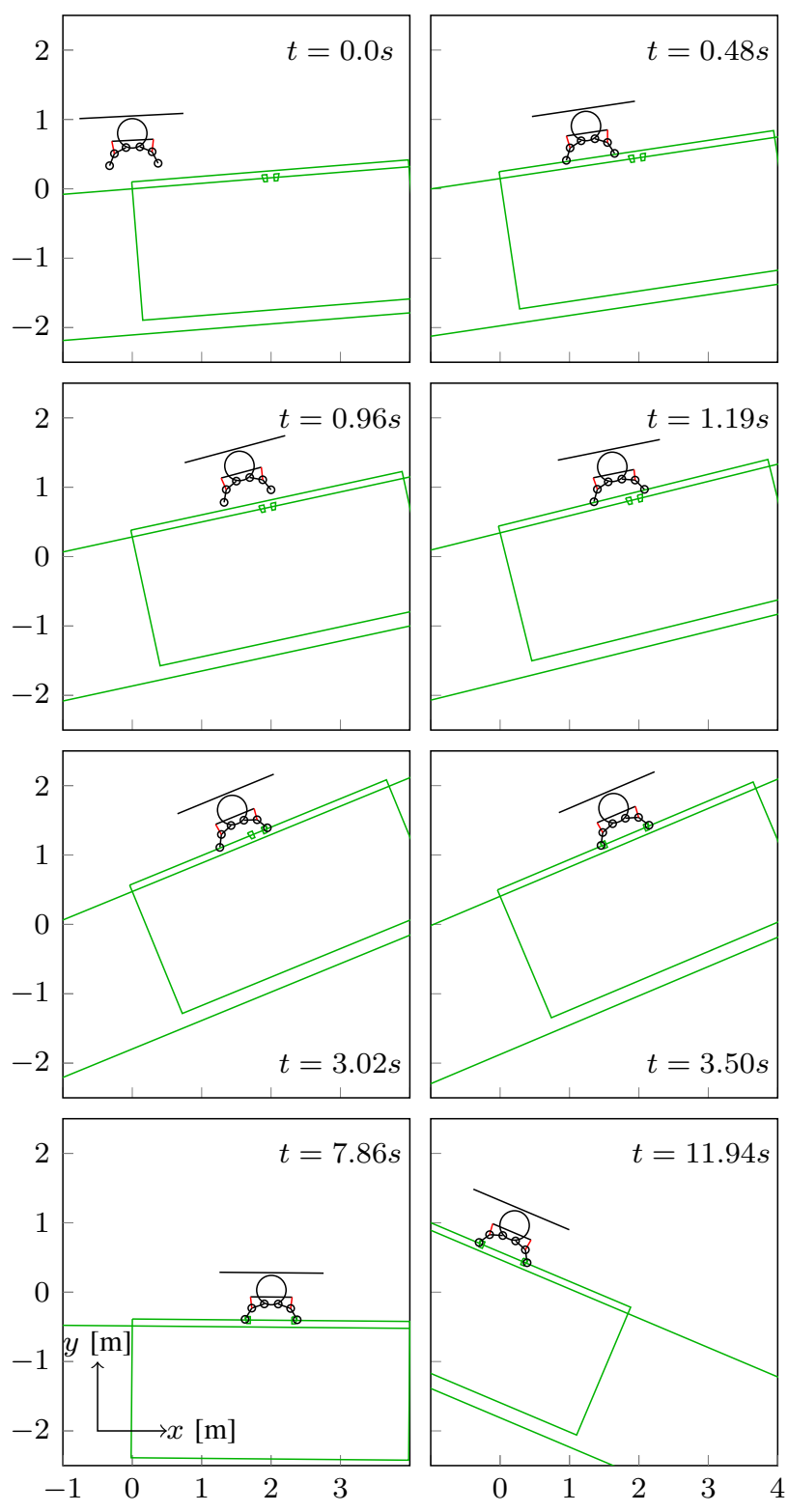

Fig. 14: (Case 5) Dynamic landing and securing simulation animation at significant timestamps 